\title{
Steady state incompressible flows using explicit schemes with an optimal local preconditioning
}

\author{
M. Storti ${ }^{* .1}$, N. Nigro ${ }^{2}$, S. Idelsohn ${ }^{1}$ \\ Grupo de Tecnologia Mecinica del INTEC, Universidad Nacional del Litoral and CONICET, Güemes 3450, 3000, Santa Fe, \\ Argentina
}

Recived 14 February 1994

\begin{abstract}
Solving large systems of equations from CFD problems by the explicit pseudo-temporal scheme requires a very low amount of memory and is highly parallelizable, but the CPU time largely depends on the conditioning of the system. Fur advective systems it is shown that the rate of convergence depends on a condition number defined as the ratio of the maximum and the minimum group velocities of the continuum system. If the objective is to reach the steady state, the iemporal term can be modified in order to reduce this condition number. Another possibility consists in the addition of a local preconditioning mass matrix. In this paper an optimal preconditioning for incompressible flow is presented, also applicabic io compressibie ones with locally incompressible zones, like stagnation points, in contrast with the artificial compressibility method. The preconditioned system has a rate of convergence independent from Mach number. Moreover. the discrete solution is highly improved, eliminating spurious oscillations frequently encountered in incompressible flows.
\end{abstract}

\section{Notation and symbols}

\section{Notation}

$\boldsymbol{x}, \boldsymbol{X} \quad$ Vectors in lower case, matrices in upper case

$\dot{x}$

$\operatorname{Re}\{z\}, \operatorname{Im}\{z\} \quad \operatorname{Real}$ and imaginary part of complex number $z$

$x_{i} y_{i}$ $(\mathrm{d} x / \mathrm{d} t)$, temporal derivative

$\operatorname{diag}\{a, b, c, \ldots\}$

$\sum_{i} x_{i} y_{i}$, repeated indices summation convention

\section{Symbols}

$\boldsymbol{A}^{ \pm}$

$\boldsymbol{B}_{0 . t}$

$\boldsymbol{B}_{0 . t}^{\prime}$

C

Diagonal matrix with diagonal entries $a, b, c, \ldots$

\section{$c$}

Jacobian matrix of the fluxes for the $i$ th spatial direction

Absolute value of the right- and left-going waves in Section 2.2

Boundary condition matrices

Boundary condition matrices in the eigencomponent basis

Courant number, non-dimensional time step

(1) Speed of sound

(2) Artificial speed of sound for the artificial compressibilit; method

\footnotetext{
* Corresponding author.

1 CONICET Research Staff members.

${ }^{2}$ Research Fellow from CONICET.
} 


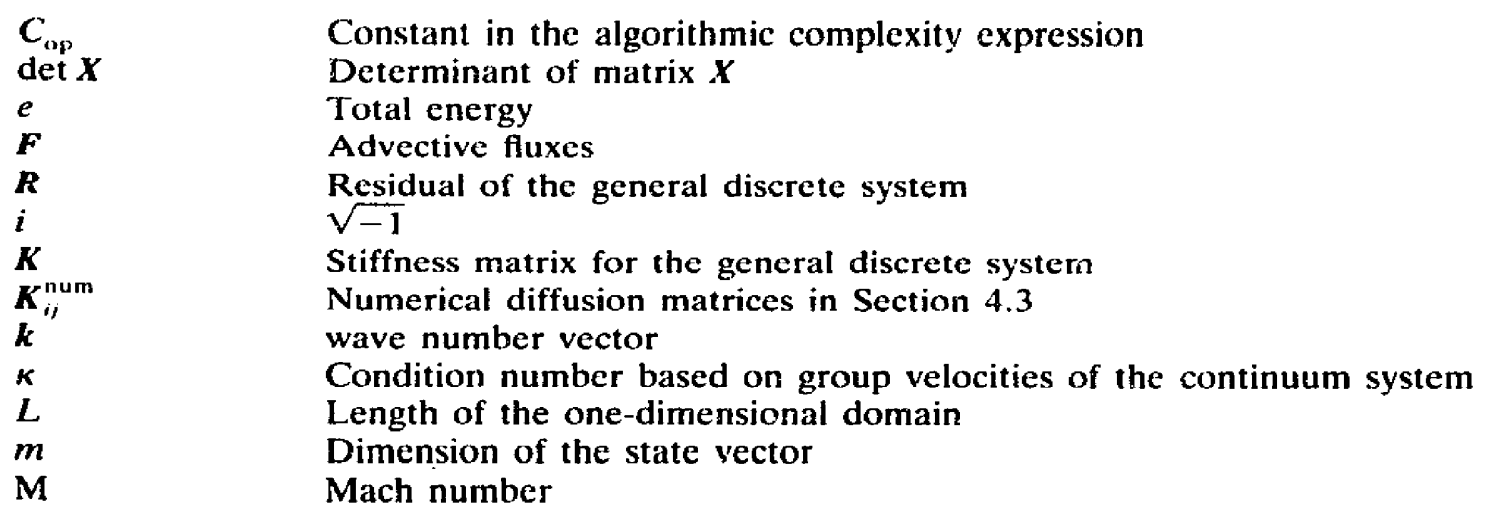

M (1) Míass matrix for the general discrete system (1)

\section{(2) Local preconditioning mass matrix for hyperbolic systems (20) \\ Artificial Mach number \\ Diagonal elements of $\boldsymbol{M}_{\text {inc }}$ \\ Number of nodes in a characteristic direction \\ Number of spatial dimensions \\ Number of degrees of freedom}

Pressure

Algorithmic constant in artificial compressibility method

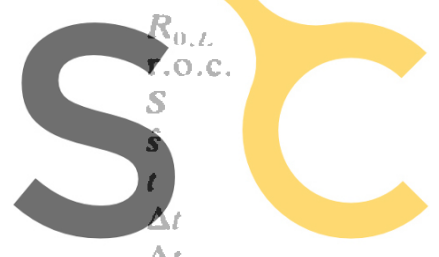

Refiection coefficients

Rate of convergence in iter
Change of basis matrix
Streamline olsented unit
Time
Time step
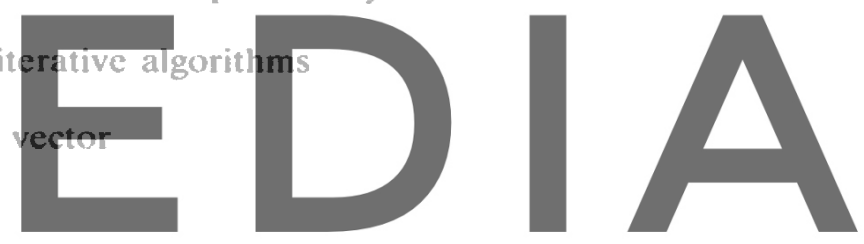

Critical time step

Register for free at https local fuid state vector

$u^{*}$
$\boldsymbol{x}$
$\boldsymbol{\alpha}$

Mach number vector

Phase velocity vector defined by $(23)$
Group velocity vector defined by (24)

$\boldsymbol{x} \quad$ State vector of the general discrete system

$\alpha \quad$ (1) Dummy scalar constant in the definition of homogeneous function

(2) Constant to adjust in the definition of $\boldsymbol{M}_{\text {inc }}$, Eqs. (46) and (49)

(3) Angle of attack in Section 5

$\begin{array}{ll}\beta & \text { Scalar parameter for hyperbolic system (57) } \\ \kappa & \text { Condition number } \\ \tilde{\kappa} & \text { Conditioning of the preconditioned system } \\ \boldsymbol{A} & \text { Jacobians in diagonal form } \\ \hat{\lambda} & \text { Eigenvalue } \\ \boldsymbol{\sigma} & \text { Angle formed by the Mach vector with the mesh } \\ \rho & \text { Density } \\ \rho_{0} & \text { Constant density in artificial compressibility method } \\ \omega & \text { Eigenfrequency for the plane-wave analysis }\end{array}$

Sub-and Supra-indices

$i, j, l$

inc

L1

L2
Spatial coordinates indices

Preconditioning based on diagonal scaling for incompressible flow

Preconditioning based on the $L_{1}$-norm of the jacobian flux vector

Same for the $L_{2}$-norm 
num

$\mathrm{C}, \mathrm{NC}$

$1 \mathrm{D}$

$\mu$

$\boldsymbol{s}$

For the numerical diffusion terms

For the conservative and non-conservative versions of the artificial compressitility system

Optimal one-dimensional values tor the numerical diffusion operator of SUPG

State vector coniponent index $(1 \leqslant \mu \leqslant m)$

Preconditioning based on the projection of the jacobian flux vector onto the streamline direction

\section{Introduction}

A very common procedure in order to find steady states from non-linear equations arising in computational fiuid dynamics, say $\boldsymbol{F}(\boldsymbol{x})=\mathbf{0}$, is to iterate an explicit or implicit temporal scheme until convergence: $\boldsymbol{M} \dot{\boldsymbol{x}}=\boldsymbol{F}(\boldsymbol{x}), t \rightarrow \infty[4,6]$. Here, $\boldsymbol{x} \in \mathbb{R}^{N}$ is the state vector, $\boldsymbol{F}$ is a map from $\mathbb{R}^{N}$ onto itself that represents the discrece system of equations, $M$ is the mass matrix of the system and the dot represents time derivative. If an explicit scheme is used, comparatively low anount of core memory and large CPU-time are required. Moreover, the CPU-time highly depends on the conditioning of the system. Bad conditioning of the system is caused by several multiplicative factors like large variations in element size through the mesh, large variations in edge sizes for a given element. local incumpressible $(\mathrm{M} \rightarrow 0)$ or transonic $(\mathrm{M} \rightarrow 1)$ behavior, where $\mathrm{M}$ is the Mach number. On the other hand, if an implicit temporal scheme coupled to a direct solver is preferred, very large convergence rates are achieved, but it requires a large amount of memory to factorize the associated matrix. This problem can be partially overcome by solving the linear subproblems by an iterative solver like GMRES, DMR, etc... The mount of core memory lighly depends on the cond implicit) is, then, related arising from large variations in mesh element siz tepping' strategies. This can be sden as modifying
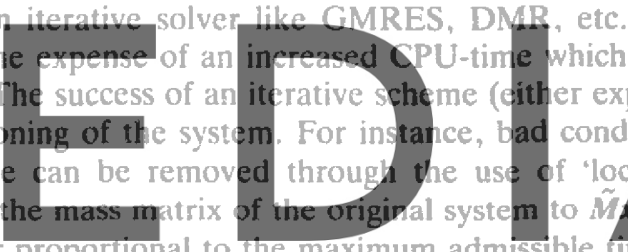

base $M$ is a diagonal matrix that includes a factor proportonal to the maxmum admisible time step based on a local stability analysis. As is well known, this modifies the ternporal evolution of the state

looking for a steady state. Much in the same way, we look for local mass matrices which correct the bad conditioning associated to different characteristic speeds at incompressible $(\mathrm{M} \rightarrow 0)$ and transonic $(\mathrm{M} \rightarrow 1)$ regimes. By 'local' mass matrices we mean block-diagonal matrices with each block connecting only the d.o.f.'s of each node. This restriction is imposed in order to have an $\mathrm{O}\left(N_{\text {nod }}\right)$ cost per preconditioning cycle, where $N_{\text {nod }}$ is the total number of nodes in the mesh.

In [7], we showed that the bad conditioning arising at transonic and incompiessible regimes is caused by very different propagation speeds for the different components of error. For instance, in the compressible regime, vorticity waves (i.e. shear waves) propagate downstream with the velocity of the fluid, which is much lower than the speed of sound with which pressure waves propagate in all directions. Thus, the limiting component in order to fulfill the CFL condition are the pressure waves, which will propagate at, roughly, one element per time step. On the other hand, the vorticity waves will propagate at $M$ elements per time step, where $M$ is the Mach number. As the main mechanism of convergence is the absorption of perturbations at the boundaries, the convergence rate will be affected by a factor $\kappa=\left|\boldsymbol{v}_{\mathrm{G}}\right|_{\max } /\left|\boldsymbol{v}_{\mathrm{G}}\right|_{\text {max.min }}$ where $\left|\boldsymbol{v}_{\mathrm{G}}\right|_{\max \text { min }}$ are the maximum and minimum group velocities. $\boldsymbol{\kappa}$ is nained the group velocity condition number and we have $\kappa=1 / M$ for incompressible flows and $\kappa=2 /|\mathrm{M}-1|$ for transonic flow. Note that $\kappa$ is a property of the continuum PDE's sysiem regardless of the numerical scheme.

In the same paper, we presented a preconditioning mass matrix (PMM) for the transonic regime. An important improvement of the conditioning of the system was reached. However, no improvement was found for the incompressible regime. On the other hand. the incompressible range is very important, perhaps even more important than the transonic one since it is present in globally compressible flows at stagnation points, for instance. 
Several general strategies can be adopted to propose PMM's. For diagonalizable systems the optimal choice is $\boldsymbol{M}=|\boldsymbol{A}|$. Here 'optimal' means that the group velocity condition number of the preconditioned system is $\mathrm{O}(1)$. Unyortunaiely, this is not the case for the gas dynamics equations. However, this choice gives some improvement in the transonic case, but fails in the incompressible one.

The preconditioning proposed here for the incompressible regime is based on a totally different concept. The idea is to rescale the momentum and continuity equations as a function of Mach number with respect to the energy equation. Both theoretical and numerical results show that an optimal conditioning is achieved $(\kappa \rightarrow 2$ for $M \rightarrow 0)$.

In Section 2 we define a condition number based on group velocities for hyperbolic systems and we show how it is related to the rate of convergence. In Section 3 we review the results already published about the application of PMM's to compressible flow at transonic regime. In Section 4 the new PMM for incompressible flow is proposed and the resulting conditioning is assessed. We devote Subsection 4.2 to the comparison with the artificial compressibility method of Chorin, while the influence on the accuracy of the numerical results is discussed in Section 4.3. Finally, in Section 5 we present several numerical results.

\section{Convergence rate in explicit pseudo-temporal sinemes}

\subsection{General considerations about rate of convergence in iterative systems}

Let us consider the explicit pseudo-temporal scheme applied to a linear system of O.D.E.'s like
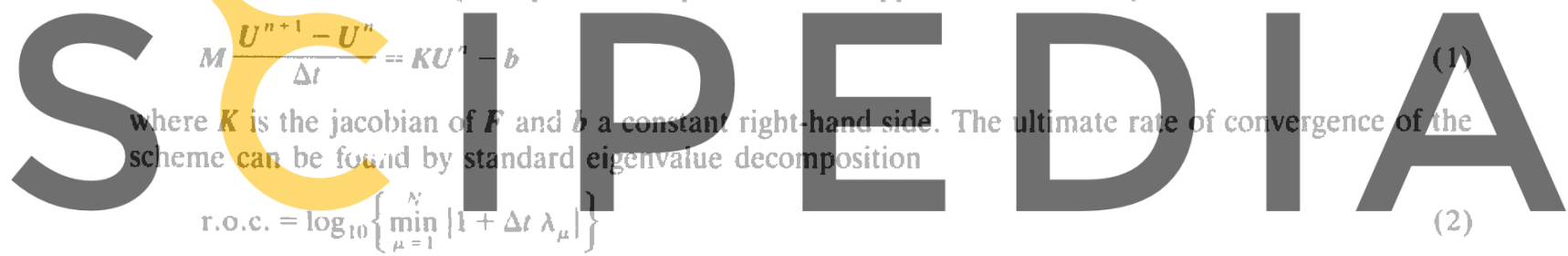

(2)

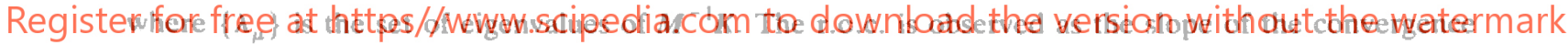

history of the residual as a function of iteration number in a logarithmic plot, measured in orders of magnitude per iteration. As is well known, snch an explicit scheme has a critical time step $\Delta l_{\text {car }}$ due to stability restrictions. For badly-conditioned systems, the largest eigenvalue $|\lambda|_{\text {wax }}$ fixes the critical time step, roughly $\Delta T_{\text {crit }} \times 1 /|\lambda|_{\max }$, and the iowest eigenvalue gives the lowest convergence rate

$$
\text { r.o.c. } \approx \log _{10}\left\{1+\Delta i|\lambda|_{\min }\right\}=\log _{10}\left\{1+\frac{|\lambda|_{\text {min }}}{|\lambda|_{\text {max }}}\right\}=\log _{10}\left\{1+1 / \kappa_{\text {disc }}\right\} \approx \frac{1}{\kappa_{\text {disc }}}
$$

where $\kappa_{\text {disc }}=|\lambda|_{\min } /|\lambda|_{\max }$ is the cordition number of the discrete system. For discretized advection systems, there are two main mechanisms of convergence, namely, numerical damping within the domain and advection to the boundaries followed by absorption. It was shown in [7] that the first one gives a convergence rate $\mathrm{O}\left(N^{2}\right)$ iterations, where $N$ is the number of elements in a characteristic direction, whereas the second one gives an $\mathrm{O}(N)$ convergence rate. As usual, the highly oscillatory modes are the stability limiting ones, whereas the smooth ones have the lowest convergence rates.

\subsection{One-dimensional example}

Now we will present a simple example that allows us to understand the main factors affecting convergence rate in advective systems. The analysis will be restricted to the continuum system since, as was mentioned, the lowest rates are those of the smoother modes. We consider a one-dimensional, linear and homogeneous system like

$$
\frac{\partial U}{\partial t}+A \frac{\partial U}{\partial x}=0,0<x<L, 0<t<x
$$


where $U \in \mathbb{R}^{2}$ is the state vector and $A \in \mathbb{R}^{2 \times 2}$ is the advective flux jacobian. We suppose that $A$ has two eigenvalues $\left\{a^{+},-a^{-}\right\}$, with $a^{2}>0$, and the boundary and initial conditions are

$$
\begin{aligned}
& B_{i} U(0, t)=0 \\
& B_{t} U(L, t)=0 \\
& U(x, 0)=U_{t}(x)
\end{aligned}
$$

The $B$ 's are $1 \times 2$ ntatrices since, as dictated by the theory of advective systems, we have one ingoing and one ouigoing wave at each boundary. Convergence rates can be detsminied by Laplace transform but, in practice, it is equivalent to seatch for solutions of the form: $U(x, t)=e^{-\lambda t} \hat{U}(x)$, and arrive at an equation for $\lambda$. Replacing this particular form in (4)

$$
-\lambda \hat{U}+A \frac{\partial \hat{U}}{\partial x}=0
$$

whose solution is

$$
\hat{U}(x)=\exp \left\{\lambda A^{-1} x\right\} \hat{U}(0)
$$

On the other hand, the boundary conditions are transformed to

$$
\begin{aligned}
& \boldsymbol{B}_{0} \hat{U}(0)=0 \\
& \boldsymbol{B}_{L} \hat{U}(L)=B_{L} \exp \left\{\lambda A^{-1} L\right\} \hat{U}(0)=0
\end{aligned}
$$

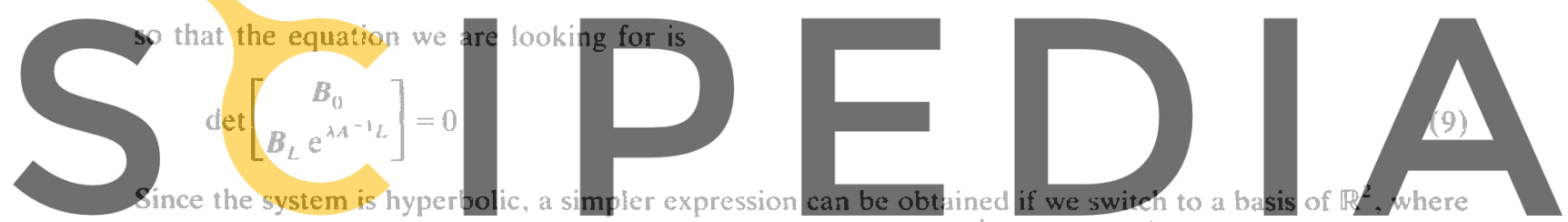

$\mathbb{A}$ is diagonal. Let $S$ be the change of basis matrix such that $S^{-1} A S=\operatorname{diag}\left\{a^{+},-a^{-}\right\}$, then

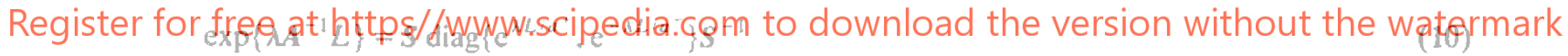

where diag $\{a, b, c \ldots\}$ stands for a diagonal matrix with diatgonal entries $a, b, c \ldots$ Transforming the boundary conditions into the eigencomponents

$$
\begin{aligned}
& B_{0}^{\prime}=B_{0} S \\
& B_{L}^{\prime}=B_{L} S
\end{aligned}
$$

and (9) simplifies to

$$
\operatorname{det}\left[\begin{array}{cc}
B_{01}^{\prime} & B_{02}^{\prime} \\
B_{L 1}^{\prime} \mathrm{e}^{\lambda L^{\prime} a^{*}} & B_{L 2}^{\prime} \mathrm{e}^{-\lambda t: a t}
\end{array}\right]=0
$$

It can be shown that the $B_{i,}$ 's are related to the reflection coefficients $R_{i l .2}$ at the extremes of the domain through

$$
R_{0}=\frac{B_{02}^{\prime}}{B_{01}^{\prime}}, \quad R_{L}=\frac{B_{L I}^{\prime}}{B_{L 2}^{\prime}}
$$

and then, Eq. (11) becomes

$$
\mathrm{e}^{-\lambda L / a^{-}}-R_{0} R_{L} \mathrm{e}^{\lambda L / a^{*}}=0
$$

from which the following expression for $\lambda$ can be obtained 

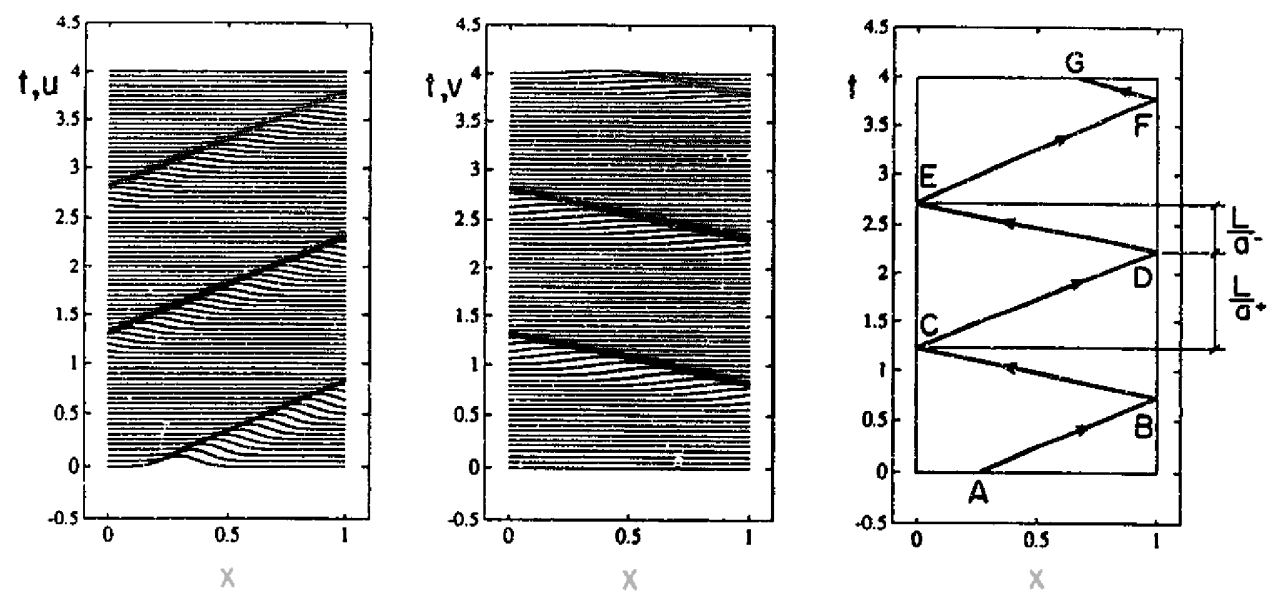

Fig. 1. Convergence by absorption at the boundaries. $L=1, a^{+}=1, a^{-}=2$. The coefficients of reflection are $R_{0,1}=+0.8$ at each boundary. The perturbation at $t=0$ is a right-geing gaussian of width $=0.1$ and unit amplitude in $u$ centered at $x=0.3$. It propagates to the right and hits the right boundary at $t=0.7$. It is reflected to a left-going $v$-wave of amplitude 0.8 tuntil it hits the left-boundary at $t=1.2$. It is reflected there to a right-going 1 -wave of amplitude 0.64 . At $t=1.5$ it is at the same place where the process started and the cycle restarts again with a toal loss of amplitude in the cycle of $\left|R_{t} R_{t}\right|$.
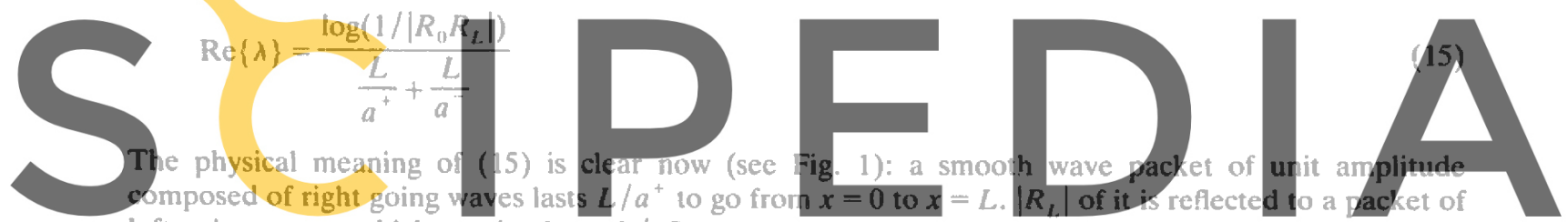

left-going waves which, again, lasts $L / a^{-}$to traverse the domain and reach the boundary at $x=0$. Finally, after reflection $\left|R_{v} R_{L}\right|$ of the initial wave is reflected to a right-going. wave packet and the cycle

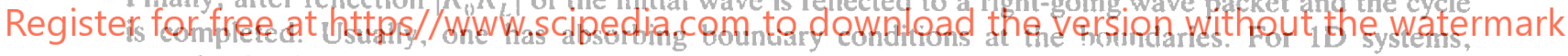

completely absorbing boundary conditions can be devised and $R_{0}, R_{\delta}=0$, implies an infinite rate of convergence. However, in practical 2D or 3D situations, local non-reflecting boundary conditions are completely absorbing only for normally incident waves and, then, a globally non-infinite rate of convergence is observed.

\subsection{Rate of convergence in advective systems}

Applying the rate of convergence definition (2) to the previously shown example, we arrive at

$$
\text { r.o.c. }=\Delta t \frac{\log _{14}\left(1 /\left|R_{0} R_{L}\right|\right)}{L\left(\frac{1}{a^{+}}+\frac{1}{a^{-}}\right)}
$$

We assumed that $\Delta t \lambda<1$ (it is shown below that it behaves like $1 / N$ as $N \rightarrow \infty$, with $N$ the number of nodes in the one-dimensional mesh). The time step is made non-dimensional: $\Delta t=C h / a_{\mathrm{mux}}$, where $C$ is the Courant number, which is restricted by the CFL condition to be smaller than unity (recail that the analysis is given for the explicit forward Euler scheme), $h$ is the mesh size and $a_{\max }=\max \left\{a^{+}, a^{-}\right\}$. Replacing the above expression for $\Delta t$ in (16) we obtain

$$
\text { r.o.c. }=\frac{C \log _{10}\left(1 /\left|R_{0} R_{L}\right|\right)}{N(1+\kappa)}
$$

with 


$$
\kappa=\frac{a_{\max }}{a_{\min }}=\frac{\max \left\{a^{+}, a^{-}\right\}}{\min \left\{a^{+}, a^{-}\right\}}
$$

This rate of convergence is valid also in multidimensional problems, where now $N$ is the number of elements in a characteristic direction, i.e. $N \sim N_{\text {nadd }}^{1, n_{d}}$. One easily arrives at an estimate of the algorithmic complexity of the form

$$
N_{\mathrm{op}}=\text { number of operations }=C_{\mathrm{up}} N_{\mathrm{rud}}^{1+1 / n_{\mathrm{s}}}
$$

The exponent is competitive with most iterative solvers and moreover, the explicit scheme is highly parallelizable and easy to code. However, one of the main drawbacks of this and most iterative solvers is the high dependence of $C_{o p}$ on the different physical and numerical parameters of the problem. For instance, very low r.o.c.'s are obtained if badly designed boundary conditiois (i.e. non-absorbing ones) are used, or the continuum system is badly conditioned ( $\kappa$ large) as in the incompressible or transonic regimes.

In the rest of the work we will focus on how to improve the r.0.c."s based on the design of at good local preconditioning. This is done in two steps: the first one is to improve the condition number $\kappa$ based only on an analysis of the continuum system. Once a good cancidate is found, it must be verified how the corresponding critical Courant number is modified, since it is ircluded also in the expression for the r.o.c. (see Eq. (16)). This is performed by a standard stability analysis. There exist cases where a given preconditioning improves the conditioning of the system but, after a stability analysis, it is shown that this 'gain' is counteracted by a deterioration in the critical Courant number so that no overall gain is produced.
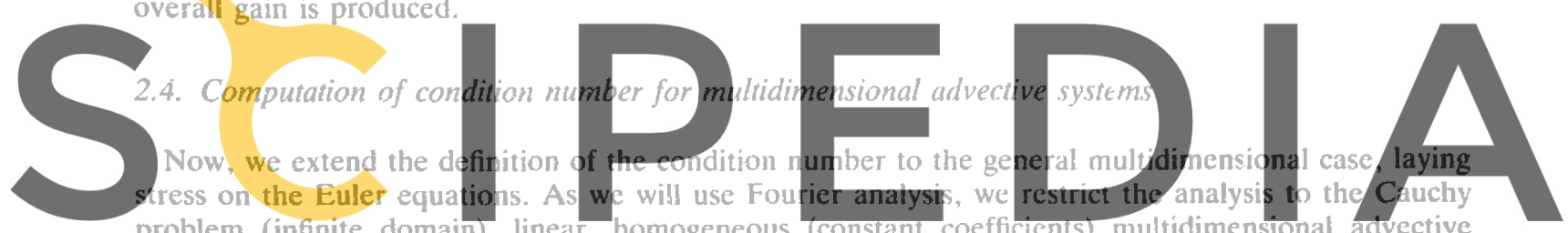

problem (infinite domain), linear, homogeneous (constant coefficients) multidimensionali advective

systems of equations

Register for freg at https//www.scipedia.com to download the version without the watermark $M \frac{\partial U}{\partial t}+A_{j} \frac{\partial U}{\partial x_{j}}=0, \quad \forall x \in \mathbb{R}^{n_{d}}, i>0$

where $U(x, t) \in \mathbb{R}^{m}$ is the state vector, $\left\{\boldsymbol{A}_{j}\right\}_{j=1}^{n_{d}}$ are the jacobians of the advective fluxes, $\boldsymbol{A}_{j} \in \mathbb{R}^{m \times m}$, and $M \in \mathbb{R}^{m \times m}$ is the mass matrix. Here and in what follows, the Einstein convention will be adopted. We assume that the system is hyperbolic, i.e. for all $k \in \mathbb{R}^{n_{d} d}$ the matrix $k \cdot \boldsymbol{A}=k_{j} \boldsymbol{A}_{j}$ is diagonalizable with real eigenvalues. Moreover, we will assume that the system is symmetrizable, i.e. there exists a non-singular matrix $S$, independent of $k$, for which $S(k \cdot A) S^{-1}$ is symmetric for all $k$. The Euler equations fall into both categories. We look for eigenfunctions in the form of plane waves

$$
U(x, t)=\hat{U} \mathrm{e}^{i(k \cdot x-a r t)}
$$

Replacing (21) in (20) the following determinantal equation in $\omega$ is obtained

$$
\operatorname{det}\left(-\omega M+k_{j} A_{j}\right)=0
$$

and it results that $\hat{U}$ has to be an eigenvector corresponding to the eigenvalue $\omega$. For each $k \in \mathbb{R}^{n_{d l}}$ we obtain a set of eigenvalues $\left\{\omega_{\mu}(k)\right\}_{\mu=1}^{m}$, which are called the 'branches of eigenvalues'. For the non-preconditioned system $M=I$, the eigenvalues are ieal since the system is hyperbolic. This feature must be kept by the preconditioning. For symmetrizable systems, a sufficient condition is that $M$ must be positive definite and symmetric in the basis where the jacobians are symmetric. Coming back to the expression for the plane wave $(21)$, we can see that constan: aimplitude planes $\operatorname{Re}\left\{\hat{U} \mathrm{e}^{\mathrm{i}(\boldsymbol{k} \cdot \boldsymbol{x}-\omega t)}\right\}=$ constant, have a characteristic phase velocity 


$$
\boldsymbol{v}_{\phi \mu}(k)=\frac{\omega_{\mu}}{k^{2}} k
$$

However, it can be shown that energy and information propagate at the group velocity

$$
\boldsymbol{v}_{\mathrm{k \mu}}=\frac{\partial \omega_{\mu}}{\partial \boldsymbol{k}}
$$

For a complete description on Fourier analysis of discrete systems the reader is referred to [9]. It is easy to see that the $\omega_{i:}$ 's are homogeneous functions of degree one in $k$, i.e. $\omega_{\mu}(\alpha k)=\alpha \omega_{\mu}(k)$ for $\alpha>0$. As a consequence, $v_{\xi \mu}$ is homogeneous of degree 0 and then it depends only on the direction of $k$

$$
v_{g \mu}(k)=v_{g \mu}\left(\frac{k}{k}\right)
$$

The condition number $\kappa$ is extended to the multidimensional case as

$$
\kappa=\frac{\left|v_{g \mu}\right|_{\max }}{\left|v_{g \mu}\right|_{\min }}
$$

where the maximum and minimum are taken over all $|k|=1$ and $\mu=1, \ldots, m$.

\subsection{Numerical computation of group velocities}

A first attempt to compute group velocities is to replace the derivatives in Eq. (24) by finite quotients, but often the cigenvalues are computed with numetical routines and some kind of continuation algorithm should be applied to identify the continuous branches. Moreover, singularities exist in some cases. We present here a practical algorithm to compute then, that overcomes this drawback. In this section the Einstein convention of summation over Let us consider the eigenvalue decomposition of $\Sigma, k_{r} A$,

$$
A=S^{-1}\left(\sum k_{j} A_{j}\right) S
$$

Register for free at https//www.scipedia.com to download the version without the watermark with $\boldsymbol{\Lambda}$ a diagonal matrix. Of course. $\boldsymbol{\Lambda}$ and $S$ are functions of $k$. By its definition, the $\omega_{\mu}$ 's are the diagonal elements of $\Lambda$

$$
\omega_{\mu}=\left\{S^{-1}\left(\sum_{j} k_{j} A_{j}\right) S\right\}_{\mu \mu}
$$

The group velocity is, by (24)

$$
v_{g \mu l}=\frac{\partial \omega_{\mu}}{\partial k_{l}}=\left\{S^{-1} A_{l} S+D\right\}_{\mu \mu}
$$

where

$$
D=\left(\frac{\partial}{\partial k_{l}} S^{-1}\right)\left(\sum_{l} k_{i} A_{i}\right) S+S^{-1}\left(\sum_{l} k_{i} A_{i}\right) \frac{\partial S}{\partial k_{l}}
$$

Taking derivatives of the identity $S^{-1} S=I$ the expression

$$
\left(\frac{\partial}{\partial k_{l}} S^{-1}\right) S+S^{-1} \frac{\partial S}{\partial k_{l}}=0
$$

is obtained, from which $\left(\partial S^{-1} / \partial k_{t}\right)$ can be eliminated and replaced in the expression for $D$ and, after some algebra

$$
D=-\left(S^{-1} \frac{\partial S}{\partial k_{l}}\right) \boldsymbol{A}+\boldsymbol{\Lambda}\left(S^{-1} \frac{\partial S}{\partial k_{l}}\right)=-E \boldsymbol{A}+\boldsymbol{A} E
$$


Now, we note that the diagonal elements of $D$ are null

$$
D_{\mu \mu}=-E_{\mu \mu} \omega_{\mu}+\omega_{\mu} E_{\mu \mu}=0
$$

and then, the group velocities can be easily computed as

$$
v_{g \mu l}=\frac{\partial \omega_{\mu}}{\partial k_{l}}=\left\{S^{-1} A_{l} S\right\}_{\mu \mu}
$$

The numerical procedure consists in sweeping the circle (sphere) $|k|=1$ in $2 \mathrm{D}$ (3D) with a large amount of wave number vectors and to compute the maximum and minimum group velocities (in absolute value). Sometimes it is interesting to plot the locus of the group velocities in a $\left(v_{g x}, v_{g y}\right)$ plane.

\section{PMM for compressible flows (review)}

The compressible Euler equations in conservative form are

$$
\frac{\partial U}{\partial t}+\frac{\partial F_{t}}{\partial x_{t}}=0
$$

where

$U=\left[\begin{array}{c}\rho \\ \rho u \\ \rho e\end{array}\right]$
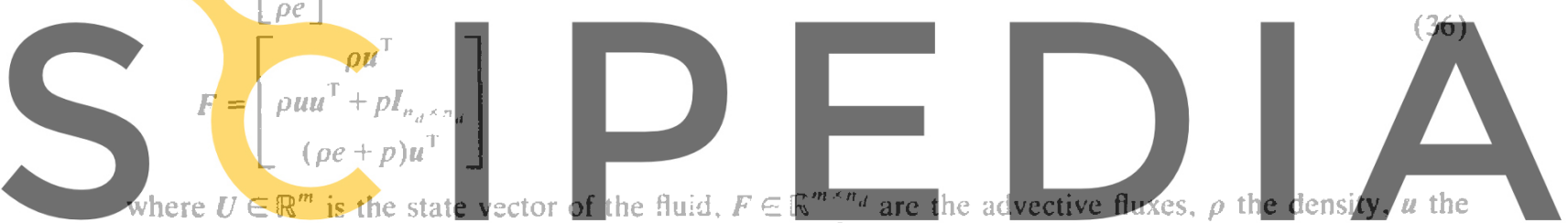

velocity vector, $p$ the pressure, $e=p /|\rho(\gamma-1)|+u^{2} / 2$ the total energy, $\gamma=1.4$ and $m=5$ in $3 \mathrm{D}$

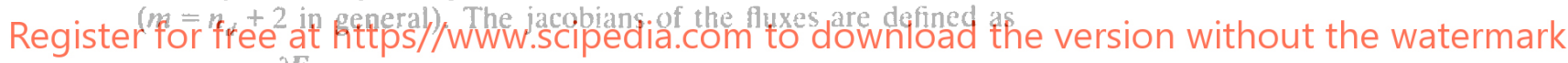

$$
A_{1}=\frac{\partial F_{i}}{\partial U}
$$

and can be written in compact form as

$$
k_{i} \boldsymbol{A}_{t}=\left[\begin{array}{ccc}
0 & \boldsymbol{k}^{\mathrm{T}} & 0 \\
-(\boldsymbol{u} \cdot \boldsymbol{k}) \boldsymbol{u}+(\gamma-1) \frac{u^{2}}{2} k & u k^{\mathrm{T}}+(\boldsymbol{u} \cdot \boldsymbol{k}) \boldsymbol{I}-(\gamma-1) \boldsymbol{k} u^{\mathrm{T}} & (\gamma-1) \boldsymbol{k} \\
(\boldsymbol{u} \cdot \boldsymbol{k})\left[(\gamma-1) u^{2}-\gamma e\right] & \left(\frac{p}{\rho}+e\right) k^{\mathrm{T}}-(\gamma-1)(\boldsymbol{u} \cdot \boldsymbol{k}) u^{\mathrm{T}} & \gamma(\boldsymbol{u} \cdot \boldsymbol{k})
\end{array}\right]
$$

The system is symmetrizable and then hyperbolic, but it is not diagonalizable in the multi-dimensional case. However, it is (trivially) diagonalizable in the one-dimensional case. We will show that for such diagonalizable systems we have an optimal preconditioning.

For the one-dimensional case. the choice

$$
\boldsymbol{M}=\left|\boldsymbol{A}_{x}\right|
$$

gives a decoupled system in the basis of eigenvectors of $A$, which looks like

$$
\frac{\partial V_{\mu}}{\partial t}+\operatorname{sign}\left(v_{\mathrm{g} \mu}\right) \frac{\partial V_{\mu}}{\partial x}=0, \quad \mu=1, \ldots m
$$

where $\boldsymbol{V}=\boldsymbol{S}^{-1} \boldsymbol{U}$ are the new variables where the system is decoupled, and $v_{\mathrm{y} \mu}$ are the corresponding eigenvalues. (For such one-dimensional systems group velocities and phase velocities coincide with the eigenvalues of the jacobian matrix.) It is clear from (40) that all components will propagate with the 
same unitary speed. For multidimensional diagonalizable systems (39) can be extended as $\boldsymbol{M}=|\boldsymbol{A}|$, and several possibilities are obtained depending on the chosen norm. In this paper we will consider

$$
\begin{aligned}
& M_{L, 1}=\left|A_{1}\right|+\left|A_{y}\right|+\left|A_{z}\right| \\
& M_{L, 2}=\sqrt{A_{x}^{2}+A_{y}^{2}+A_{z}^{2}}
\end{aligned}
$$

Again, we transform the syste, into the basis where it is diagonal

$$
\frac{\partial V_{\mu}}{\partial t}+\frac{v_{g \mu i}}{\left|v_{g \mu}\right|} \frac{\partial V_{\mu}}{\partial x_{i}}=0, \quad \mu=1, \ldots, m
$$

where $v_{g \mu i}$ is the $i$ th dimensional component of the group velocity for the $\mu$ th equation of the non-preconditioned system, and it can be shown that it corresponds to the $\mu$ th diagonal component of the jacobian $A_{i}$ in its diagonal form. (For a detailed discussion see [7].) The norm appearing in (43) is the same as that one chosen for the preconditioning matrix. It stems from this expression that the group velocities for the preconditioned system are all $O(1)$.

A first attempt to treat non-diagonalizable systems is to use (41) or (42) directly. The condition number for a particular $M$ is computed as described in Section 2.4, and we plot the condition number for both the non-preconditicned system $\kappa_{\mathrm{NP}}$ (see Fig. 2) and the preconditioned ones $\kappa_{\text {L.t.i. }}$. As can be seen, some gain is sbtained for transonic flow. The gain is a constant factor as $\mathrm{M} \rightarrow 1\left(\kappa_{t, 1, t, 2} / \kappa_{\mathrm{NP}} \rightarrow 1 / 3\right.$ as $M \rightarrow 1)$, since both give a behavior $O(|M-1|)^{-1}$ as $M \rightarrow 1$. See Fig. 3 for logarithmic scaling in the transonic region.

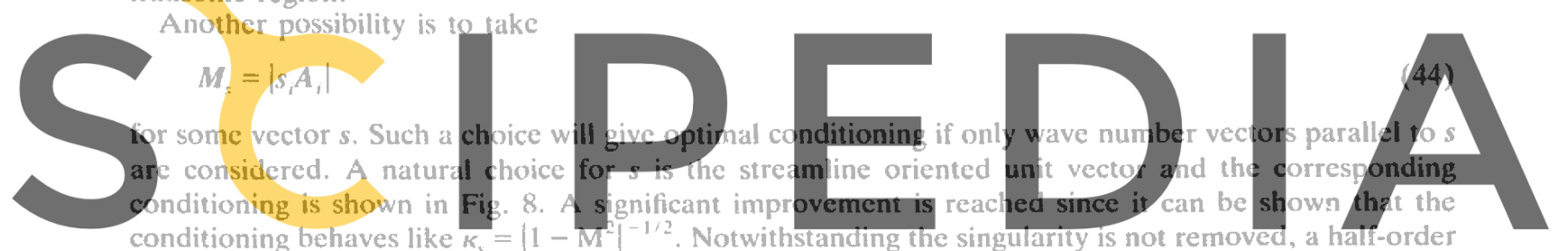
conditioning behaves like $k_{\mathrm{s}}=\left|1-\mathrm{M}^{-1}\right|^{-1 / 2}$. Notwithstanding the singularity is not removed, a half-order gain is achieved.

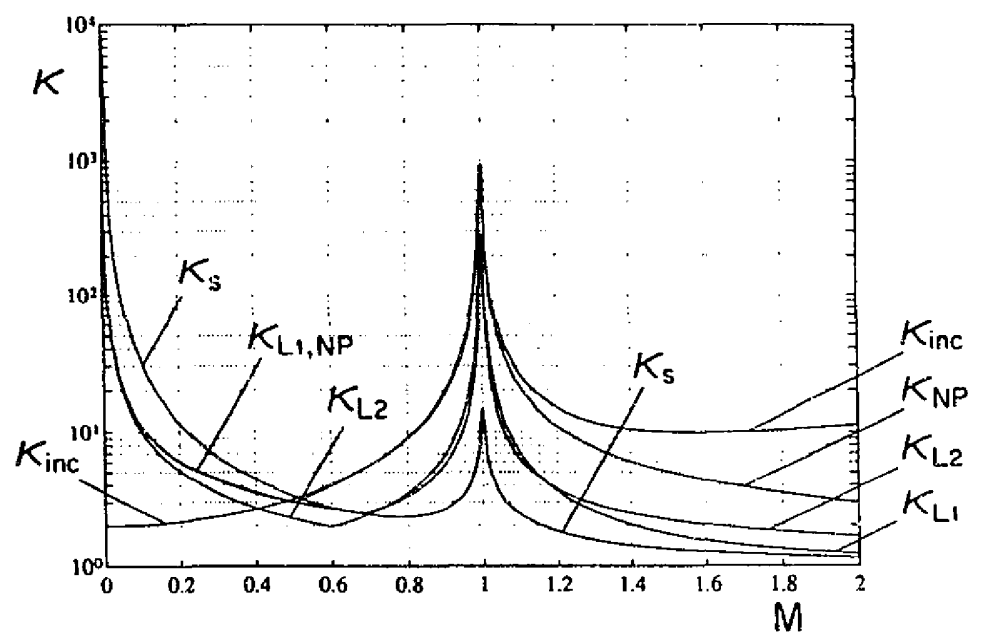

Fig. 2. Semi-logarifhrne plot of the condition number of the continuum system for several preconditionings. 


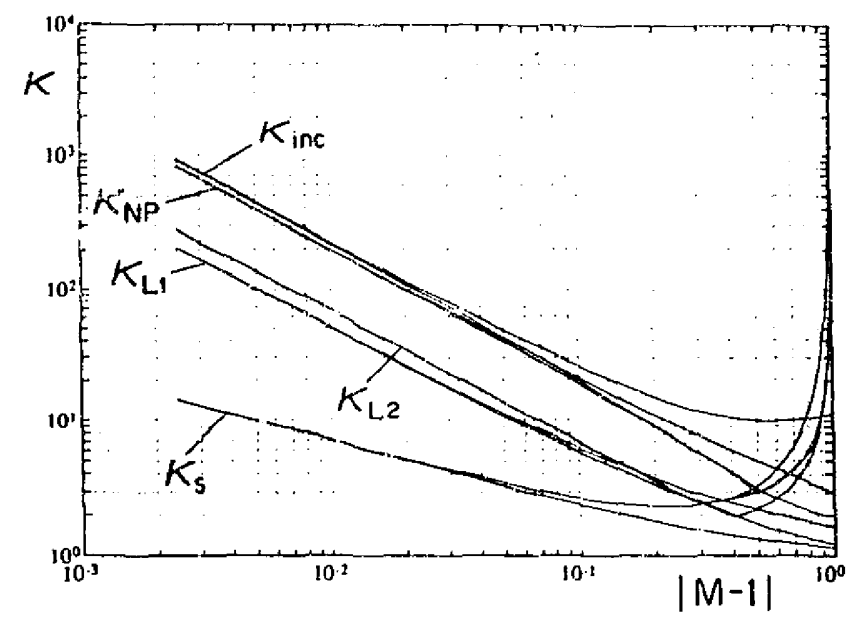

Fig. 3. Same as Fig. 2, but with al logarithmic scaling in the transonic regime $\mathbf{M} \rightarrow 1$.

\section{PMM for incompressible flows}

For the incompressible regime, none of the above-mentioned possibilitics succeeded in giving a significant improvement in the conditioning of the system. See Fig. 4 for logarithmic scaling in the incompressible region. In fact, the PMM's from Eqs. (41) or (42) have a condition number $O(1)$ as $\boldsymbol{M} \rightarrow 0$, so that it is clear that it cannot correct the singularity. Regarding the streamline based $\boldsymbol{M}_{s}$ from Eq. (44), the conditioning is even worse than the non-preconditioned system. In Figs. 5-8 we can see the locus of the group velocity vector in the $\left(v_{\mathrm{ky},}, v_{\mathrm{gy}}\right)$ plane for $\mathrm{M}=0.1$ to 0.6 with $\Delta \mathrm{M}=0.1$ and $\boldsymbol{M}=\boldsymbol{I}$, $M_{L 1, L 2, s}$ As is well known, for the non-preconditioned system the locus for the branches corresponding to pressure waves are circles of unit radius centered at $(\mathrm{M}, 0)$. The entire branch for advection of vorticity is collapsed in a point at $(\mathrm{M}, 0)$. For the $L_{1}$ and $L_{2}$ norm the circles are deformed in quasi-circles with a nose pointing to $(1,0)$ and the advection of vorticity expands into a triangle which

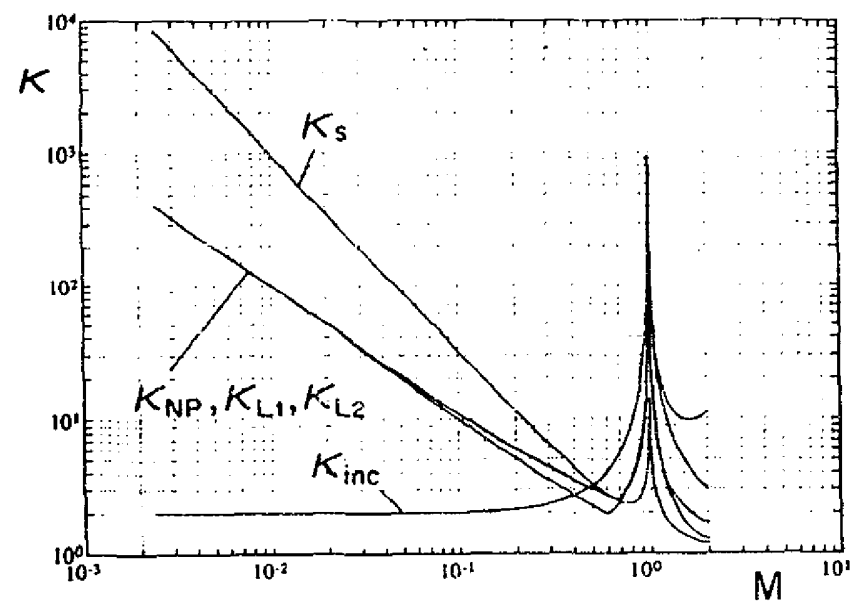

Fig. 4. Same as Fig. 2. but with a iogarithmic scaling in the incompressible regime $M \rightarrow 0$. 


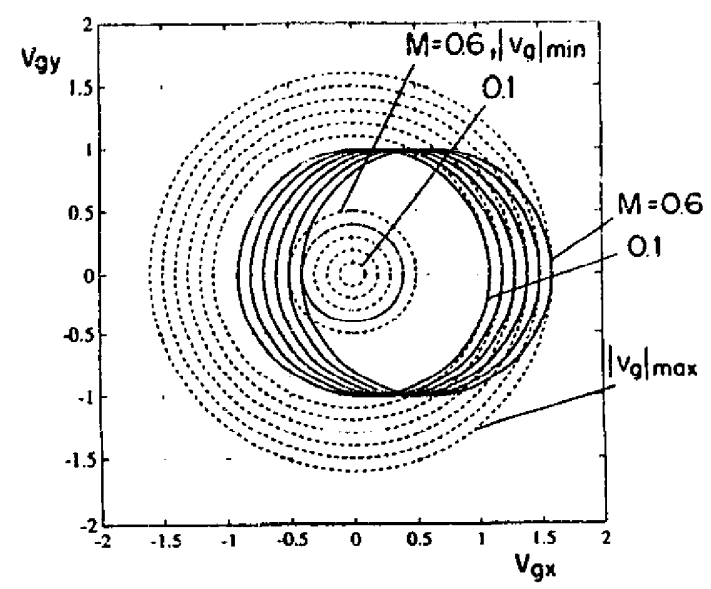

Fig. 5. Locus of group velocities for the non-preconditioned system $M=1$. The locus has been plotted for $M=0.1$ to 0.6 with a step of 0.1 . The branches of group velocities are indicated by points. For each Mach number two circles centered at the origin and with radii $|\boldsymbol{v}|_{\text {ma. } \operatorname{mon}}$ have been drawn.
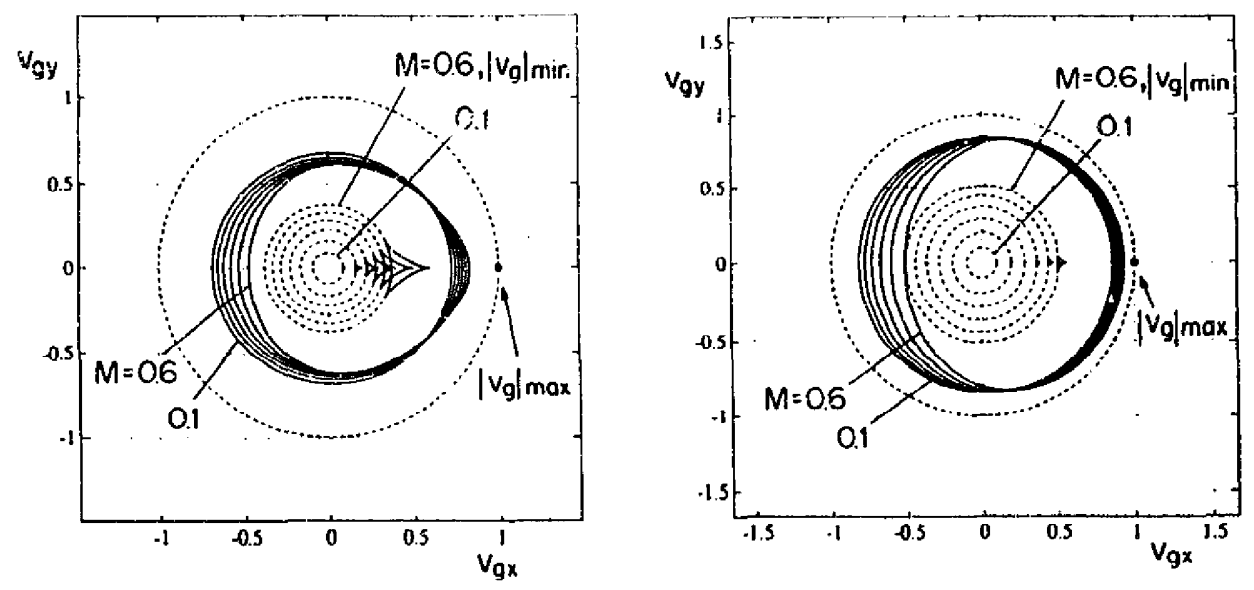

Fig. 6. Sane as Fig. 5 but for $M=M_{t, 1}$.

Fig. 7. Same as Fig. 5 but for $\boldsymbol{M}=\boldsymbol{M}_{1:}$.

diminishes in size as $M \rightarrow 0$ and a single point at $(1,0)$. For the streamline based preconditioning (44), the advection-of-vorticity branch expands in a triangle and collapses with the quasi-circle resulting in a cardioid-shaped curve and a single point at $(1,0)$.

\subsection{Preconditioning by diagonal scaling}

As was mentioned in the Introduction, the preconditioning we propose for the incompressible case here is based on 'tuning' three different scaling parameters for the continuity. momentum and energy equations. For the sake of simplicity we perform an analysis for the $2 \mathrm{D}$ Euler equations in primitive variables. The jacobians are

$$
k_{i} A_{i}=u_{i} I+\left[\begin{array}{ccc}
0 & \rho k^{\mathrm{T}} & 0 \\
0 & 0_{n_{d} \times n_{d}} & k / \rho \\
0 & \rho c^{2} k^{\mathrm{T}} & 0
\end{array}\right]
$$




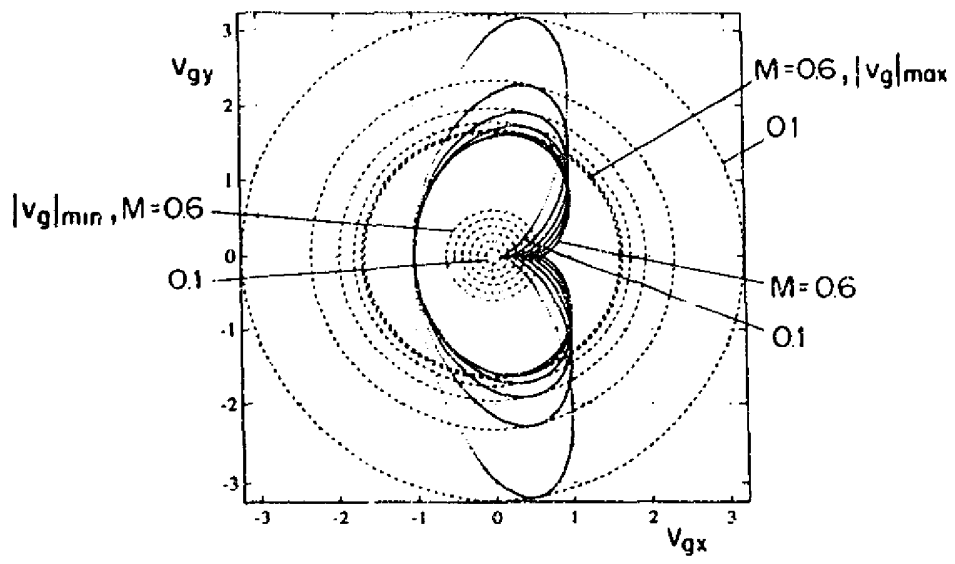

Fig. 8 . Same as Fig. 5 but for $M=M_{2}$.

Without loss of generality, we will suppose that $u$ is parallel to the $x$ axis. The proposed mass matrix is

$$
\boldsymbol{M}_{\text {inc }}=\operatorname{diag}\{m 1, m 2, m 2, m 3\}
$$

These parameters will be chosen in such a way so as to improve, as far as possible, the conditioning for the one-dimensional problem when $k_{y}=0$. It is expected that, if the conditioning works in this case, it will work for the general $k_{y} \neq 0$ case, since the proposed matrix (46) is isotropic. This result is confirmed afterwards.

The group velocities for $k_{y}=0$ have only $x$ components (due to symmetiy about the $x$-axis), which is obtained through the resoluition of the following eigenproblem

$$
\operatorname{det}\left(A_{x}-v_{g x} M\right)=0
$$

whose solution is, in the limit of low Mach numbers

$$
v_{\mathrm{gx}}=\left\{\begin{array}{l}
\mathrm{M} / m_{1} \\
\mathrm{M} / m_{2} \\
\frac{\mathrm{M}\left(m_{2}+m_{3}\right)}{2 m_{2} m_{3}} \pm \sqrt{\left[\frac{\mathrm{M}\left(m_{2}+m_{3}\right)}{2 m_{2} m_{3}}\right]^{2}+\frac{\left(1-\mathrm{M}^{2}\right)}{m_{2} m_{3}}} \approx \pm\left(m_{2} m_{3}\right)^{-1 / 2}
\end{array}\right.
$$

In order to have a $O(1)$ condition we must have

$$
\frac{\mathrm{M}}{m_{1}}=\frac{\mathrm{M}}{m_{2}}=\alpha^{2}\left(m_{2} m_{3}\right)^{-1 / 2}
$$

with $\alpha$ a constant independent from Mach number. Since the mass matrix is defined up to a multiplicative constant, we can set $m_{1}=\alpha \mathrm{M}$ and then, $m_{2}=m_{1}=\alpha \mathrm{M}$ and $m_{3}=1 / \mathrm{M}$ are obtained from (49).

Now we proceed to the 'fine' tuning of the preconditioning. i.e. the choice of the optimal value for $\alpha$. This is done by sweeping $\alpha$ for a fixed Mach number and plotting the condition number versus $\alpha$. The optimal value $\alpha^{*}(\mathrm{M})$ which gives the lowest condition number is found and it can be seen that $\alpha^{*} \rightarrow 2$ when $\mathbf{M} \rightarrow 0$ (see Fig. 10). The final expression for $\boldsymbol{M}_{\text {inc }}$ is

$$
\boldsymbol{M}_{\text {inc }}=\operatorname{diag}\left\{2 \mathrm{M}, 2 \mathrm{M}, 2 \mathrm{M}, \frac{1}{\mathrm{M}}\right\}
$$

The locus of group velocities for $M=0.1$ to 0.6 is shown in Fig. 9. Note that both circles at the maximum and minimum group velocities approach a defined value as $M \rightarrow 0$, so that the condition number remains bounded. 

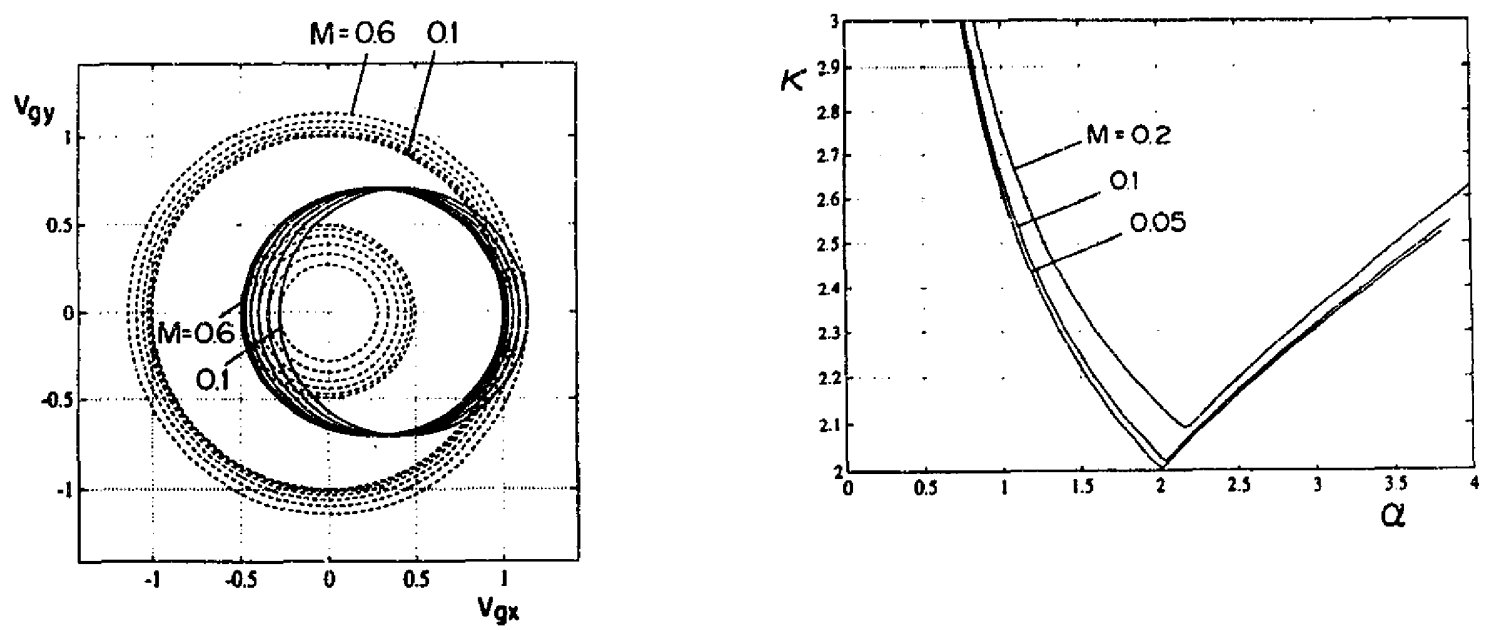

Fig. 9. Same as Fig. 5 but for $\boldsymbol{M}=\boldsymbol{M}_{\mathrm{tni}}$.

Fig. 10. Optimal value for the $\alpha$ constant. Condition number of the preconditioned system $\left(M=M_{17 c}\right.$. Eq. (50)) as a function of $\alpha$ for $\mathrm{M}=0.05,0.1,0.2$.

As was mentioned in the last paragraph of Section 2.3, we checked the stability of the discretized preconditioned system. The integration scheme is the standard forward-Euler. The stability analysis is done by Fourier analysis of the infinite problem. Stability is assessed for each plane wave corresponding to a wave number vector $k=\left(k_{x}, k_{y}\right)$. For each Mach vector $u^{*}=u / c=\left(u_{x}^{*}, u_{y}^{*}\right)$ we sweep a number of representative wave number vectors and the maximum admissible time step $\Delta l\left(u^{*}, k, \mu\right)$ is computed (for a detailed description see [7]). We are interested in the Courant number associated to the above critical time step. The maximum admissible Courant number is calculated as

$$
C_{\operatorname{mix}}=\frac{\left|v_{g}\right|_{\max }}{h} \min _{k, \mu} \Delta t\left(u^{*}, k, \mu\right)
$$

In Fig. 11 we plot the Courant number versus the incidence angle $\theta=\tan ^{-1}\left(u_{y}^{*} / u_{x}^{*}\right)$ for several $\left|u^{*}\right|$ and for both the preconditioned (right) and non-preconditioned (left) cases. We conclude that the stability limits are not drastically affected by the preconditioning.

Regarding the cost of this preconditioning, it is negligible as compared to the cost of the evaluation of the residual. It amounts mainly to the multiplication of the residual of each equation by the inverse of the corresponding diagonal element in the preconditioning matrix. This multiplication must be performed for each node, at update time.

\subsection{Comparison with the artificial compressibility method}

The preconditioning proposed in Section 4.1 has several aspects in conımon with the artificial compressibility method of Chorin, and in this section we will analyze the advantages and similarities with it. The 'artificial compressibility equations' can be cast as an advective diffusive system $(35,36)$ with the following definitions

$$
U=\left[\begin{array}{l}
p \\
u
\end{array}\right], \quad F_{\mathrm{C}}=\left[\begin{array}{c}
\rho_{i j} c^{2} u \\
u u^{\mathrm{T}}+p I_{n_{d} \times n_{d}}
\end{array}\right], \quad A_{\mathrm{C}^{2}} k_{i}=\left[\begin{array}{cc}
0 & c^{2} k^{\mathrm{T}} \\
k & u_{\mathrm{i}} I+u k^{\mathrm{T}}
\end{array}\right]
$$

$c^{2}$ is a positive parameter that can be chosen in such a way so as the improve the convergence to the steady state. In [5], a local value of $c^{2}=\max \left(0.3, r u^{2}\right)$ with $1<r<5$ is suggested.

We performed an analysis of the conditioning of the system based on the ratio between the maximum 

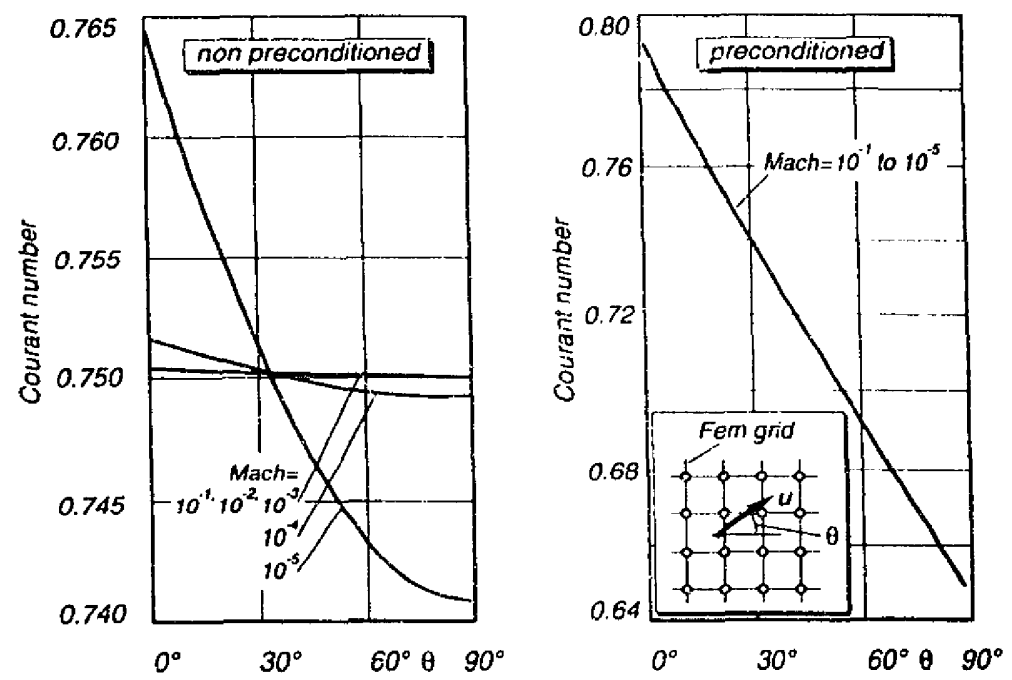

Fig. 11. Stability analysis for the non-preconcitioned (left) and preconditioned (right) systems. We have the critical Courant number (based on $\|+c$ ) on the ordinates and angle of the flow with the mesh axis in the abscissac. Each curve corresponds to a different Mach number for $\mathrm{M}=10^{\prime}, 10^{2}, \ldots, 10^{\text {". }}$

and minimum group velocities, and a sharper estimation of the parameter was obtained. We consider the linearized (i.e. with constant jacobians) version of (52) and we note that the condition of the system depends only on the 'artificial' Mach number $M_{a r}=u / c$. The lowest condition number is obtained for $M_{a n t}=1 / \sqrt{3}$ and the corresponding condition number is $\kappa=3$. The corresponding locus of group velocities is shown in Fig. 12. $\mathbf{M}_{\text {art }}=1 / \sqrt{3}$ corresponds to an $r$ value of 3 .

Instead, a small improvement is obtained if the following form of the artificial compressibility equations is considered
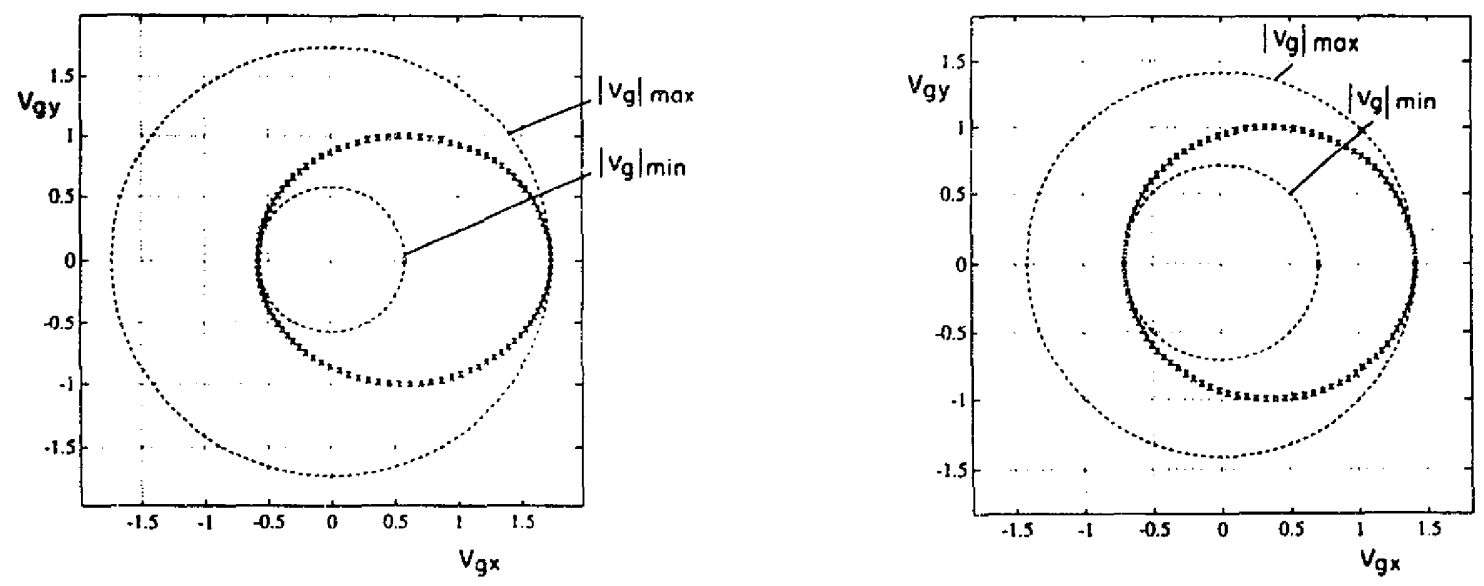

Fig. 12. Locus of group velocities for the artificial compressibility equations in conservative form (52). The crosses indicate the branch of group velocitics and two circles centered at $v_{g}=0$ of radii $\left|v_{2}\right|_{n a .} .\left|v_{k}\right|_{n+n}$ have been drawn. The figure corresponds to $\mathbf{M}_{\mathrm{an1}}=1 / \sqrt{3}$.

Fig. 13. The same as in Fig. 12 for the non-conservative form of the antificial compressibility ecuations $(53)$, for $M_{a r t}=1 / \sqrt{2}$. 


$$
A_{\mathrm{NC} i} k_{i}=\left[\begin{array}{cc}
0 & c^{2} k^{\mathrm{T}} \\
k & u_{i} I
\end{array}\right]
$$

This system is obtained if a term proportional to the continuity equation is substracted from the momentum equations. However, the conservative character of the equations is lost. The lowesi condition number is obtained at $M_{a r t}=1 / \sqrt{2}$ and is $\kappa=2$ (see Fig. 13), exactly the same as in our proposed scheme.

Finally, if the conservative form of the equations is used together with a PMM as follows

$$
\boldsymbol{M} \frac{\partial U}{\partial t}+\frac{\partial \boldsymbol{F}_{\mathrm{C} i}}{\partial x_{i}}, \quad \boldsymbol{M}=\left[\begin{array}{cc}
1 & \mathbf{0}_{n_{d} \times n_{d}} \\
i k / c^{i} & I
\end{array}\right]
$$

the same value of $\kappa=2$ at $M_{\text {art }}=1 / \sqrt{2}$ is obtained (see Fig. 14), but in a conservative form now.

The preceding results led to the following conclusions. First, both the artificial compressibility method in conservative form of Eq. (52) and the PMM of Section 4.1 give optimal (i.e. $\kappa=O(1)$ ) conditionings with a slight advantage for the PMM $(\kappa=2$ against 3$)$. The PMM is applicable to incompressible flows (it suffices to consider an arbitrarily low Mach number) but also to compressible flows with incompressible regions, whereas the artificial compressibility applies only to incompressible flows. Both are almost equivalent if an incompressible flow is considered and either the nonconservative version (53) or the preconditioned conservative one (54) is considered.

\subsection{Accuracy improvement}

Another topic to be discussed is how the preconditioning affects the accuracy of the solution. First of all, we have to remark that preconditioning and discretization do not commute and, then, the discrete solution for the steady state of the preconditioned system is not the same as for the non-preconditioned one

$$
\boldsymbol{U}_{\mathrm{inc}}(t=x, h) \neq \boldsymbol{U}_{\mathrm{NP}}(t=x, h)
$$

but

$$
\lim _{h \rightarrow 0} U_{\mathrm{inc}}(t=\infty, h)=\lim _{h \rightarrow 0} U_{\mathrm{NP}}(t=x, h)=\bar{U}(t=x)
$$

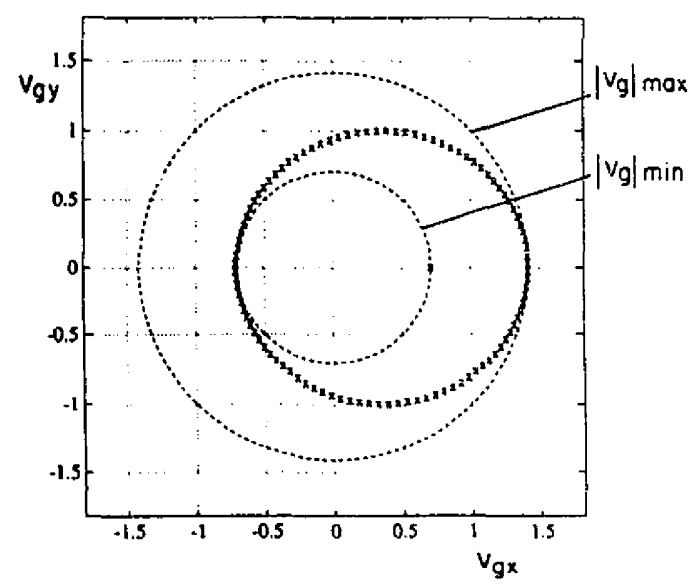

Fig. 14. The same as in Fig. 12 for the conservative form of the artificial compressibility equations with preconditioning (54). for $M_{a+1}=1 / \sqrt{2}$ 
where $\bar{U}$ is the continuous solution of the non-preconditioned problem, whereas $U_{\text {inc.NP }}$ stand for the discrete solutions of the preconditioned and non-preconditioned problems. Of course, both converge to the continuum steady solutions as the mesh is refined. The non-equivalence of the steady state discrete solutions is due to the fact that the stabilizing 'upwind' terms (in cur case, taken from SUPG) are not the same. The question that arises is whether the numerical solution is improved or deteriorated by the preconditioning. It is well known that accuracy problems can arise in nearly incompressible flows. The subject has been extensively studied in the context of Navier-Stokes equations. For the Euler equations, the problem has received much lesser attention because it is not so used to solve incompressible inviscid flows with this system. However. 'checkerboard' type instabilities have been reported in stagnation points [1].

Surprisingly enough, we have experimentally found that the numerical solutions are much improved when this preconditioning is used, as will be reported in the numerical results. We do not have a full explanation of this phenomenon, yet. At fist sight, it seems reasonable that the stabilization scheme will work better on a well-conditioned problem. Consider, for instance, the following system

$$
\frac{\partial \boldsymbol{U}}{\partial t}+\boldsymbol{A}_{i} \frac{\partial \boldsymbol{U}}{\partial x_{i}}=0, \quad \boldsymbol{U}=\left[\begin{array}{l}
u_{1} \\
u_{2}
\end{array}\right], \quad \boldsymbol{A}_{x}=\left[\begin{array}{cc}
\beta & 0 \\
0 & -\beta
\end{array}\right], \quad \boldsymbol{A}_{y}=\left[\begin{array}{cc}
0 & 1 \\
1 & 0
\end{array}\right]
$$

This system is symmetric and non-diagonalizable. As usual, in an SUPG context, the stabilized system on a square homogeneous mesh of size $h$ looks like

$$
\frac{\partial U}{\partial t}+A_{i} \frac{\partial U}{\partial x_{i}}=K_{i j}^{\mathrm{num}} \frac{\partial^{2} U}{\partial x_{i} \partial x_{j}}
$$

where

$$
K_{i j}^{\text {num }}=A_{i} \tau A_{j} \text { and } t=\frac{h}{2}\left(A_{x}^{2}+A_{y}^{2}\right)^{-1 / 2}
$$

For the system described above (57) we have

$$
\tau=\frac{h}{2 \sqrt{1+\beta^{2}}} I \quad \text { and } \quad K_{x x}^{n u m}=\frac{h \beta^{2}}{2 \sqrt{1+\beta^{2}}} I, \quad K_{y y}^{\text {num }}=\frac{h}{2 \sqrt{1+\beta^{2}}} I
$$

This should be compared to the 'optimal' one-dimensional expressions

$$
K_{x x}^{\text {num. } 10}=\frac{h}{2}\left|A_{x}\right|=\frac{h}{2} \beta I, \quad K_{y y}^{n u m .1 D}=\frac{h}{2}\left|A_{y}\right|=\frac{h}{2} I
$$

For $\beta \ll 1$ we have $K_{y y}^{\text {num }}=K_{y y}^{\text {num.1D }}$ which means that the upwinding is optimal in the $y$ direction, but $K_{x x}^{\text {num }} \approx \beta K_{x x}^{\text {num,1D }} \ll K_{x x}^{n u m .1 D}$ and the numerical dissipation along the $x$-direction is insufficienti. Conversely, for $\beta \gg 1$ we have $K_{x x}^{\text {num }}=K_{x x}^{\text {num.1D }}$ but $K_{y y}^{\text {num }} \approx 1 / \beta K_{y y}^{\text {num }, 1 \mathrm{D}} \ll K_{y y}^{\text {num, } 1 \mathrm{D}}$. For $\beta=\mathrm{O}(1)$ the upwind is nearly optimal for all directions. Coincidently, the lowest conditioning (in the sense of Eq. (26)) is obtained fo $\beta=1$. This verifies the rule: 'low conditioning implies better stabilization'.

However, this rule is not applicable to diagonalizable systems, since they are always optimally stabilized, independent from the conditioning of the system.

\section{Numerical results}

Firstly, in this section we will show how the preconditioning mass matrix proposed here improves the rate of convergence of nearly incompressible flows $\left(\mathrm{M} \sim 10^{-3}\right)$ for two model problems like the circular bump and flow around a Joukowski profile.

In Fig. 16 we can see the convergence history for the circular bump (thickness $=12 \%$, see Fig. 15) with the non-preconditioned scheme (left) and the preconditioning mass matrix presented in this paper (right). We notice that a significant improvement in the rate of convergence is achieved regardless of the very low Mach number $\left(\mathrm{M}=10^{-3}\right)$, and also validate the improvement in the condition number. 


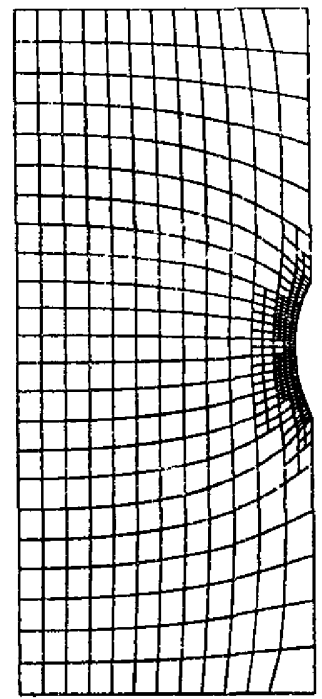

Fig. 15. Locally refined mesh for the bump (thickness $=12 \%$ ).
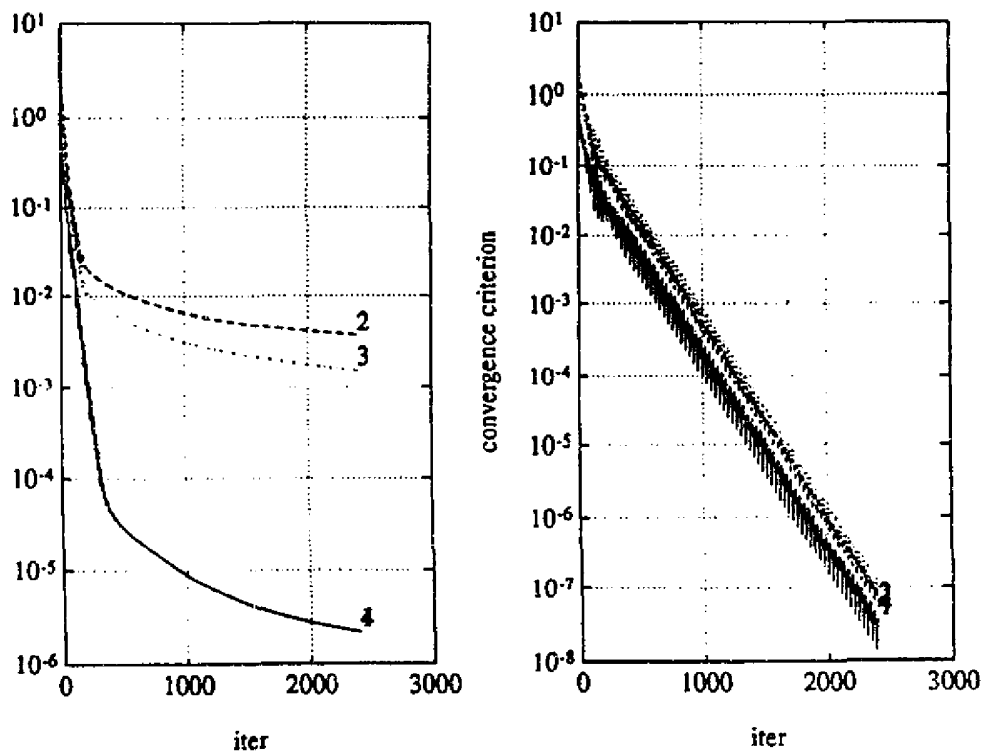

Fig. 16. Convergence history fon tin sump at $\mathrm{M}=10^{-3}$, without (left) and with (right) preconditioning. Each curve corresponds to the r.m.s.-norm of the vector of nodal residuals of each equation: 1 (continliity), $2,3(x, y$ momentum) anic $\&$ (zreroy).

Initially, for the non-preconditioned case, the residual has a relatively high r.o.c. of roughly 60 iterations/order with a highiy oscillatory component, but it switches later to a smooth cuive with a low and diminishing r.o.c. of 6000 iter/order. This behavior is explained as follows: initially the error is mainly in the continuity equation which generates pressure waves which have a high group velocity and then a high rate of convergence. The oscillations are caused by reflections at the lateral slip boundaries (absorbing boundary conditions are used at the inlet and outlet boundaries). At a certain moment the component of the error in the form of pressure waves have been dissipated, and that component in the form of vorticity remains almost with the same amplitude since it has a much lower group velocity. 

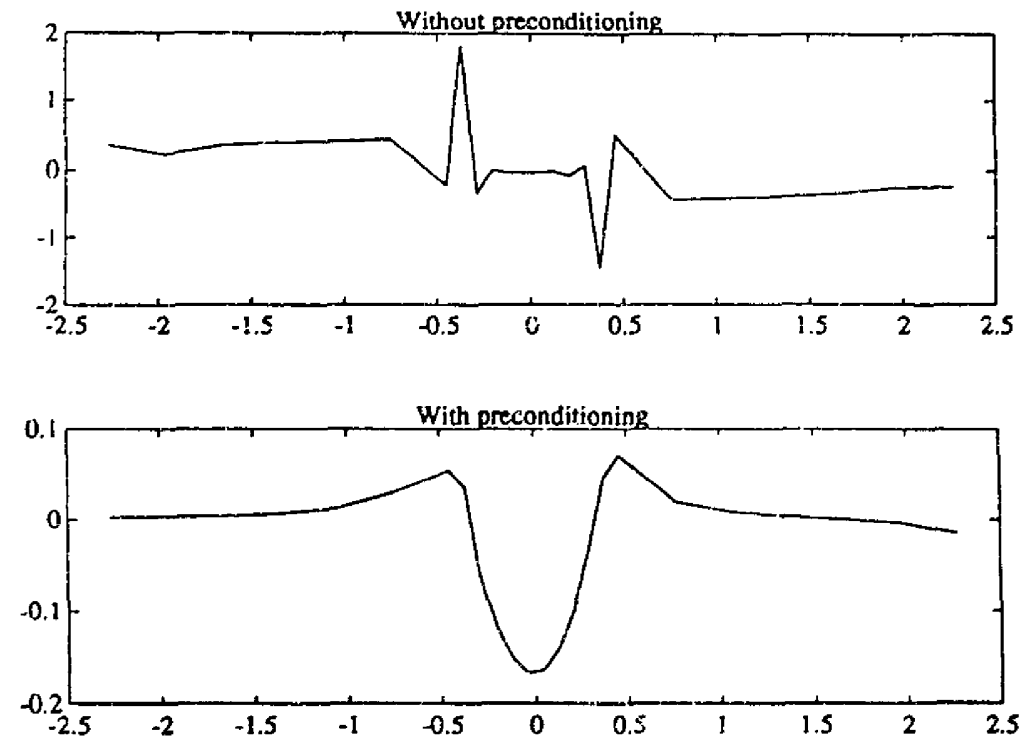

Fig. 17. $C_{r}$ distribution on the bump for the coarse mesh.

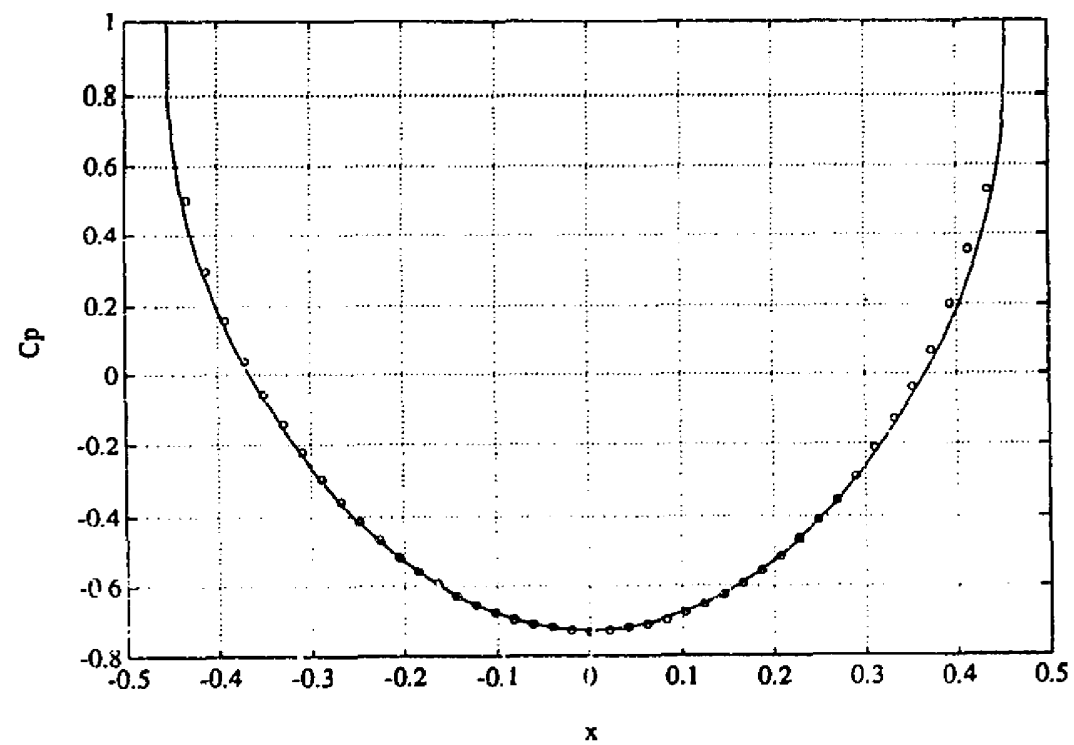

Fig. 18. $C_{p}$ distribution on the bump for the fine mesh. (O: Fuler/FEM $M=10$, 一: incompressible potential/BEM). 

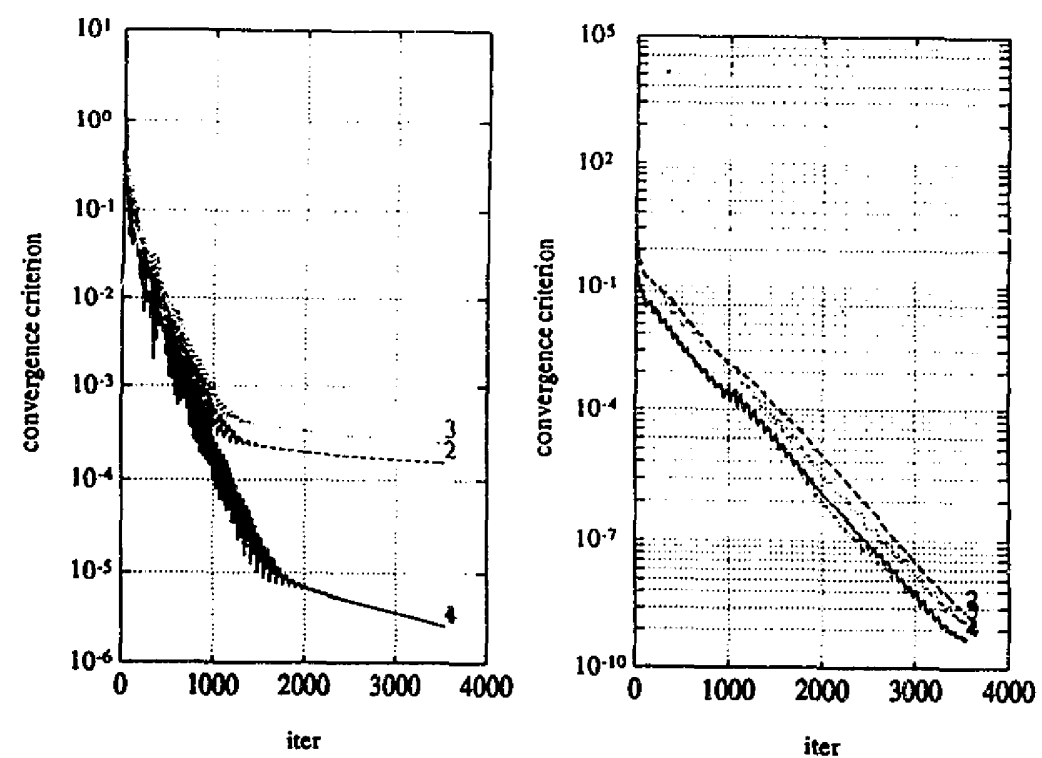

Fig. 19. Convergence history for the Joukowski profile ( $12 \%$ thickness, $4.6 \%$ camber) at an angle of attack $\alpha=-0.8872^{\circ}$ and $\mathrm{M}=10^{-3}$, without (left) and with (right) presonditioning. Labeling of the curves is the same as in Fig. 16.

From this point onwards, the r.o.c. is dominated by that of the vorticity waves, since they are the main component of the error. Since vorticity is propagated downstream, thete is $\mathrm{n}=$ possibility of reflections at the slip boundaries. This explains the smooth behavior of the second part of the curve. With respect to the preconditioned case, we can observe in the right plot that all equations have almost the same slope, and it is so because we have got a condition number close to two.

The second example is a flow around a Joukowski profile (12\% thickness, $4.6 \%$ camber) with an angle of attack $\alpha=-0.8872^{\circ}$ and a Mach number $M=10^{-3}$. As in the previous case, Fig. 19 shows the convergence histories for the non-preconditioned system (left) and for the proposed preconditioning (right) and, again, we conclude that the rate of convergence experiences an important improvement with similar rates for each equation.

Another interesting topic, treated in Sution 4.3, is the accuracy improvement. In this kind of problem one of the most important measures related to the quality of the results is got by the pressure coefficient distribution around the profile. For both problems we have a pattern to validate the numerical results. For the circular bump we have computed a pattern using a BEM (Boundary Element Method) computation for potential flow that is very close to the real experiment due to the low compressible effects. For the Joukowski profile example we have the analytic solution for incompressible potential flow, obtained from conformal mapping techniques.

In Fig. 15 we show the mesh used for the final simulation on the bump. As we can see, we have refined close to the profile to increase the accuracy. The first simulation was carried out with the basis mesh (the mesh without refinement, not shown) and the results obtained with this mesh are plotted in Fig. 17. In the upfur part we show the $C_{p}$ distribution in $y$-axis versus the profile coordinates in the $\boldsymbol{x}$-axis for the non-preconditioned scheme. The other plot is the same for the preconditioned scheme presented here. We can see how the checkerboard modes that appear in Euler codes near incompressible regimes (upper plot), disappear with the preconditioned scheme (lower plot). In order to compare to the pattern mentioned above we refined the mesh (see Fig. 15) and we present this comparison in 


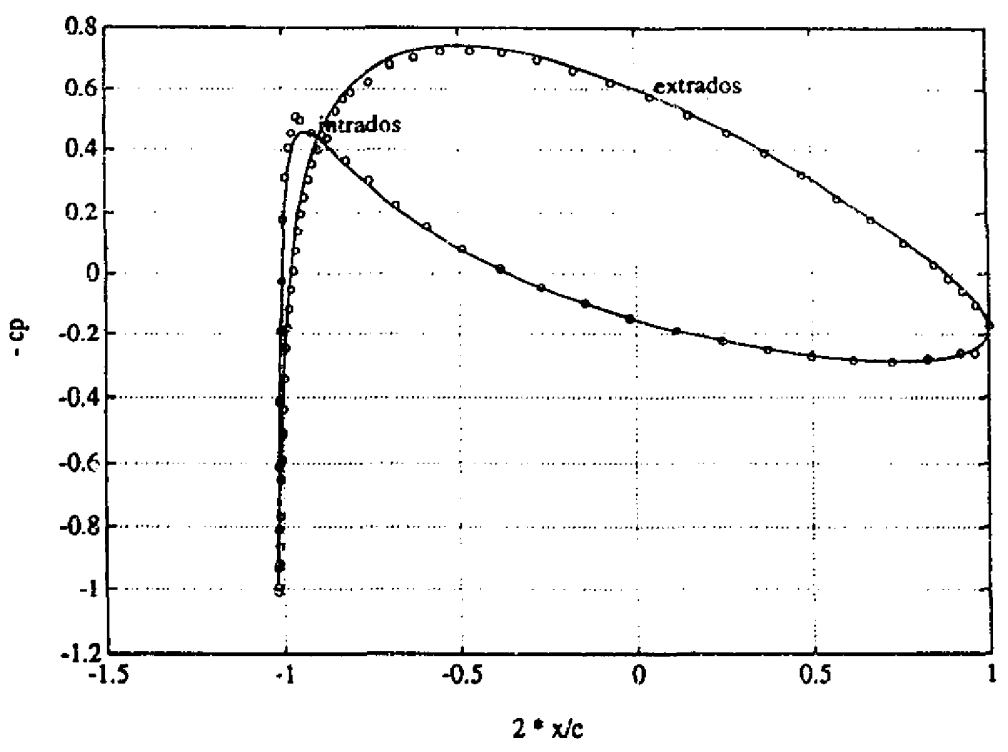

Fig. 20. $C_{p}$ distribution for the Joukowski profile. ( $O$ : Euler/FEM $M=10^{-3},-$ : cxact/incompressible potential).

Fig. 18. Note the good quality of these results even though morc refinements can be performed at the leading and trailing edge in the case that the pressure distribution has to be adjusted more precisely.

Finally, in $F: g$. 20 we show the $C_{p}$ distribution for the second example (Joukowski profile at $\mathrm{M}=10^{-3}$ with angle of attack $\alpha=-0.8872^{\circ}$ ). The numerical resuits have been marked with circles and the analytical potential flow solution in dashed line. Good agreement between both results is observed. Note also, the ability to capture the suction peak.

\section{Conclusions}

An optimal local preconditioning for incompressible flows was developed in this paper. It preserves the hyperbolicity of the system improving the rate of convergence to the steady state and the accuracy of the numerical results. As regards of the rate of convergence, this shows a behavior independent of Mach number. Unlike the artificial compressibility method of Chorin, the proposed preconditioning is applicable not only to incompressible flows, but also to compressible flows with incompressible regions. Both are almost equivalent if an incompressible flow is considered. This completes the work presented in [7], covering the whole Mach number range, from incompressible to supersonic. Such a method allows to get the goal of unification of compressible and incompressible flows. Firstly, spurious checkerboard oscillations are suppressed and, secondly, explicit schemes can be applied even at the incompressible regime.

\section{Acknowledgment}

The authors wish to express their gratitude to Consejo Nacional de Investigaciones Científicas y Técnicas (CONICET, Argentina) for its financial support. 


\section{References}

[1] M. Bristeau, R. Glowinski, L. Dutto, J. Periaux and G. Roge, Compressible viscous flow calculations using compatibles finite elements approximations, Int. J. Numer. Methods Fluids 11 (1990) 719-749.

[2] B. Gustafsson, Numerical boundary conditions, in: B. Engquist, S. Osher and R. Somerville, eds., Large-scale Cemputations in Fluid Mechanics (American Mathematical Society, Providence, 1985).

[3] G. Hauke and T.J.R. Hughes, A unified approach to compressible and incompressible flows, Comput. Methods Appl. Mech. Engrg. 113 (1994) 389-395.

[4] A. Jarneson, A non-oscillatory shock capturing scheme using flux limited dissipation, in: B. Engquist, S. Osher and R. Somerville, eds., Large-Scale Computations in Fluid Mechanics (American Mathematical Society, Providence, 1985).

[5] A. Rizzi and L. Eriksson, Computation of inviscid incompressilule flow with rotation, J. Fluid Mech. 153 (1985) $275-312$.

[6] F. Shakib and T.J.R. Hughes, A new finite element formulation for computational fluid dynamics: $X$. The compressible Euler and Navier-Stokes equations, Comput. Methods Appl. Mech. Engrg. 89 (1991) 141-219.

[7] M. Storti, C. Baumann and S. Idelsohn, A preconditioning mass matrix to accelerate the convergence to the steady Euler solutions using explicit schemes, Int. J. Numer. Methods Engrg. 34 (1992) 519-541.

[8] L. Trefethen, Stability of hyperbolic finite-difference models with one or two boundaries, in: B. Engquist, S. Osher and R. Somerville, eds., Large-scale Computations in Fluid Mechanics (American Mathematical Society, Providence, 1985).

[9] R. Vichnevetsky and J.B. Bowles, Fourier analysis of numerical approximations of hyperbolic equations, SIAM Studies Appl. Math. (1982). 